\title{
Clinical factors affecting semen improvement after microsurgical subinguinal varicocelectomy: which subfertile patients benefit from surgery?
}

\author{
Franco Palmisano (D), Daniel Moreno-Mendoza, Riccardo levoli, \\ Gabriel Veber-Moisés-Da Silva, Carlos Gasanz-Serrano, Juan Fernando \\ Villegas-Osorio, Maria Fernanda Peraza-Godoy, Álvaro Vives, Lluís Bassas, \\ Emanuele Montanari, Eduard Ruiz-Castañé, Joaquim Sarquella-Geli and \\ Josvany Sánchez-Curbelo
}

\begin{abstract}
Background: The exact mechanism of varicocele-related infertility is still elusive, therefore, the current challenges for its management lie in determining which patients stand to benefit most from surgical correction. The authors aimed to assess the clinical factors affecting semen improvement after left microsurgical subinguinal varicocelectomy (MSV) in relation to patient age, ultrasound varicocele grading (USVG), and presence of a right subclinical varicocele (RSV).

Methods: From 2010 to 2017 a total of 228 infertile patients underwent left MSV for clinical varicocele. Descriptive statistics were used to describe the cohort and verify the surgical benefit in terms of semen improvement, in addition, subsets of patients were selected according to clinical covariates. Logistic regression modeling was applied to evaluate the presence of RSV, operative time, age, and USVG as explanatory variables.

Results: Sperm concentration (SC), progressive sperm motility (PSM), and normal sperm morphology (NSM) increased significantly after surgery $(p=0.002 ; p=0.011 ; p=0.024$; respectively). Mean SC improved after MSV in $\geqslant 35$ year-old patients and the grade 3 USVG group ( $p=0.01 ; p=0.02$; respectively). Logistic regression modeling showed a that the probability of SC improvement was $76 \%$ lower in subjects presenting RSV ( $p=0.011$ ). In addition, patients with a grade 3 USVG presented a three-times greater probability of SC improvement compared with patients with a lower USVG $(p=0.035)$. In addition, older patients showed a greater probability of SC improvement after MSV ( $p=0.041)$.

Conclusions: MSV is an effective varicocele-related infertility treatment that should also be offered to older patients. In addition, patients with a higher USVG benefit from surgery. In infertile men with an RSV in association with a left clinical disease, a bilateral varicocele repair should be considered.
\end{abstract}

Keywords: age, grade, infertility, microsurgery, predictors, semen parameters, subclinical, ultrasound, varicocele, varicocelectomy

Received: 6 August 2019; accepted in revised form: 17 October 2019.

Introduction

Varicocele is defined as a pathological dilation of the testicular pampiniform venous plexus that drains the testes and may be associated with male subfertility, hypogonadism, pain, discomfort, and failure of ipsilateral testicular growth and
Ther Adv Urol

2019, Vol. 11: 1-11

DOI: $10.1177 /$

1756287219887656

(c) The Author(s), 2019 Article reuse guidelines: sagepub.com/journalspermissions
Correspondence to: Franco Palmisano Department of Urology, Foundation IRCCS Ca' Granda Ospedale Maggiore Policlinico, University of Milan, via della Commenda, Milan, Italy franco.palmisanola hotmail.it

Daniel Moreno-Mendoza Carlos Gasanz-Serrano Juan Fernando VillegasOsorio

Maria Fernanda Peraza-

Godoy

Álvaro Vives

Lluís Bassas

Eduard Ruiz-Castañé

Joaquim Sarquella-Geli

Josvany Sánchez-Curbelo

Fundació Puigvert,

Department of Andrology,

Universitat Autonoma de

Barcelona, Barcelona,

Spain

Riccardo levoli

Department of Statistics,

University of Bologna,

Bologna, Italy

Gabriel Veber-Moisés-Da Silva

Hospital de Clínicas de

Porto Alegre, Department of Urology, Porto Alegre, Brazil

Emanuele Montanari Department of Urology, Foundation IRCCS Ca' Granda Ospedale Maggiore Policlinico, University of Milan, Milan, Italy 
development. This condition is present in $11.7 \%$ of adult men and in $25.4 \%$ of men with abnormal semen analysis. ${ }^{1}$ Despite a recent meta-analysis showing semen improvement after surgical correction, the exact association between reduced male fertility and varicocele is unknown. ${ }^{2}$

Several hypotheses have attempted to explain the correlation between varicoceles and subfertility, with the most commonly acknowledged mechanisms being acceptably attributed to hypoxia and hemostasis, increased scrotal temperature, adrenal metabolite reflux, autoimmunity, and increased oxidative stress. ${ }^{3,4}$

Because the pathophysiology of varicocele-related infertility remains elusive, current challenges in its management lie in determining which patients stand to benefit most from surgical correction, and when surgery should be performed.

In this context, sparse data from the literature has led to unclear and inconsistent evidence that allows for no reliable recommendations in favor for, or against, varicocelectomy indications in the guidelines of the most important societies worldwide. In fact, the guidelines sometimes contradict each other and leave treatment indications up to the individual discussions with infertile couples. $^{5-8}$

However, current evidence indicates that the most effective treatment option is microsurgical varicocelectomy because it results in fewer complications and lower recurrence rates compared with the other techniques. ${ }^{9}$

With the purpose of identifying the optimal treatment strategy for infertile males with left clinical varicocele, the present study aims to define clinical factors affecting semen improvement after microsurgical subinguinal varicocelectomy (MSV) based on patient age, ultrasound varicocele grading (USVG), and the concomitant presence of right subclinical varicocele (RSV).

\section{Patients and methods}

We identified subfertile men with left clinical varicocele within the Department of Andrology, Fundació Puigvert, Barcelona, Spain, who underwent MSV between January 2010 and December 2017. All patients presented with at least 1 year of infertility with unprotected intercourse and had impaired semen quality according to the World Health Organization (WHO) reference values. ${ }^{10}$

The exclusion criteria were: azoospermia, concomitant right clinical varicocele or bilateral varicocelectomy, male accessory gland infection, anti-sperm antibodies, retrograde ejaculation, tumors, undescended testicle(s), and chromosome defects.

All patients underwent a detailed medical history review, and a general physical and andrological examination. Varicocele was clinically diagnosed by physical palpation and classified according to the $\mathrm{WHO}^{11}$ guidelines. In addition, an in-office testicular ultrasound (US) was performed in both the supine and standing position. Grading was assigned as follows: grade I $3 \mathrm{~mm}$ (maximum) vein diameter in the pampiniform plexus in association with reflux during the Valsalva maneuver, grade II vein diameter $>3 \mathrm{~mm}$ accompanied by reflux during the Valsalva maneuver, and grade III was spontaneous reverse vein flow that increased during the Valsalva maneuver.

The MSV procedure was performed by the same experienced surgical team using an operating microscope that allowed from $\times 6$ to $\times 25$ magnification of the operating field (Carl-Zeiss, Jena, Germany).

Intraoperative and postoperative adverse events were recorded. Follow-up was based on a standard internal protocol that consisted of a scheduled re-evaluation 7 days after surgery and an andrological evaluation with semen analysis and scrotal US 3 months postoperatively.

Semen samples were collected by masturbation directly into a sterile plastic container after 3-5 days of sexual abstinence. The following variables were taken into consideration: sperm concentration $\left(n \times 10^{6} / \mathrm{ml} \mathrm{SC}\right)$, progressive sperm motility (\% PSM) and normal sperm morphology ( $\%$ NSM).

\section{Statistical analyses}

$R$ software was used for statistical analysis $(R$ : A language and environment for statistical computing; $R$ Core Team, 2014; $R$ Foundation for Statistical Computing, Vienna, Austria). Statistical significance for the tests was set at $\alpha<0.05$. 
Descriptive and test statistics were used to describe the cohort and verify the benefits following surgery in terms of semen parameter improvements. A one-way Kolmogorov-Smirnov (KS) statistical test was applied to assess the normality of variables. Continuous variables were presented as mean \pm standard deviation (SD) and compared using a Chi-square test, while KS could not reject the null hypothesis, and the Wilcoxon signed-rank test was applied where normality could not to be assumed (KS test $p$ value $<0.05$ ). Subsequently, subsets of patients were selected according to clinical covariates and the previous analyses were repeated. A data driven approach was used to identify variables that could have potential explanatory significance for semen improvement following surgery. The variables selected were: patient age, USVG, and the presence of concomitant RSV. Regarding patient age, the cohort was divided into two groups: $<35$ year-old (group aA), and $\geqslant 35$ years old (group $\mathrm{aB}$ ), and with respect to USVG, patients were classified as 'low' (I-II, group USVG 1-2 ) or 'high' grade (III, group $\mathrm{USVG}_{3}$ ).

The main aim of the analyses was to assess and quantify the impact of covariates on semen improvement. Box plots were used to describe the analysis and to give a visual overview of the quantitative changes in semen parameters after surgery.

Finally, generalized linear model theory ${ }^{12}$ was applied to model the probability of semen improvement using some determinants. Specifically, logistic regression ${ }^{13}$ was used to model the probability of SC improvement, according to some explanatory variables, including the presence of RSV, operative time, patient age, and USVG. These variables were chosen using a selection method (stepwise).

\section{Results}

Overall, 228 subfertile patients, with a mean age of 34.1 ( \pm 6.1 ) years, underwent subinguinal microscopical varicocele repair. Clinical grade I was reported in $14.7 \%$ of patients, grade II and III were reported respectively in $50 \%$ and $35.3 \%$ of patients. The grade of clinical disease was missing for 72 patients. US staging revealed grade I for $23(12.3 \%)$ of patients, and grade II and III in $90(42.6 \%)$ and $92(45.1 \%)$ of patients, respectively, data was missing for 23 patients. US revealed grade I subclinical right varicocele in $16.23 \%$ of patients $(n=37)$. Mean operation time was $57.5 \mathrm{~min}$ ( \pm 19.9 ), all patients were discharged the day of surgery. No cases of hydrocele were reported, and 19 patients anticipated the scheduled clinical re-examination, for pain $(4.4 \%$, 10 patients), scrotal hematoma ( $2.2 \%, 5$ patients), or surgical wound discharge $(1.8 \%, 4$ patients). However, hospitalization was never necessary. Figure 1 shows significant semen parameter changes at 3 months after surgery. US persistence of varicocele was observed in 11 patients (3 grade I, 1 grade II, 7 grade III).

With regard to patient age, no statistically significant differences were found between groups aA and $\mathrm{aB}$ preoperatively (Figure 2a). Group $\mathrm{aB}$ benefited from MSV in terms of mean SC $\left(23.4 \times 10^{6} / \mathrm{ml} \pm 31.2 ; p=0.01\right)$; Figure $2 \mathrm{~b}$ shows quantitative semen changes among age groups.

According to USVG, mean age of group $\mathrm{USVG}_{1-2}$ and group $\mathrm{USVG}_{3}$ were similar, being respectively $34.7( \pm 6)$ and $33.3( \pm 6.4$ ) years old. Three months after surgery, varicocele repair resulted in a significant impact on the NSM of group $\mathrm{USVG}_{1-2}$ $(p=0.02)$, while both groups presented SC improvement ( $p=0.05$ and 0.02 respectively), as observable in Figure 3a. Figure 3b reports quantitative semen improvements after MSV at the 3-month follow-up for all US grades.

Taking into consideration the presence of RSV, a lack of improvement of semen parameters following surgery is highlighted in cases with RSV, while patients with only left disease significantly improved after MSV in terms of all considered semen parameters (Figure 4).

Table 1 shows the main results of the logistic regression model, the probability of an SC improvement (of $1 \times 10^{6} / \mathrm{ml}$ of concentration) was lower in patients presenting with a concomitant RSV (76\% lower than others, ceteris paribus; $p=0.011$ ). This probability varied from $14 \%$ to $77 \%$ in $95 \%$ of the samples. In addition, the probability of considerable improvement in terms of SC was three-times greater for patients with $\mathrm{USG}_{3}$ than for patients with $\mathrm{USG}_{1-2}$, ceteris paribus $(p=0.035)$. Moreover, the probability of SC improvement for patients with $\mathrm{USG}_{3}$ was greater for each age group of patients $(p=0.041)$, with the chances varying from $0.03 \%$ to $11 \%$ in $95 \%$ of samples. 
Table 1. Logistic regression model. Dependent variable: sperm concentration.

\begin{tabular}{lclllll}
\hline & Estimate & Standard error & Z value & OR & CI (95\%) & $p$ value \\
\hline Age & 0.053 & 0.026 & 2.048 & 1.054 & $(1.003-1.110)$ & $0.041^{*}$ \\
USVG 2 & 0.703 & 0.497 & 1.412 & 2.019 & $(0.769-5.506)$ & 0.158 \\
USVG 3 & 1.081 & 0.511 & 2.114 & 2.946 & $(1.095-8.267)$ & $0.035^{*}$ \\
RSV & -1.090 & 0.431 & -2.531 & 0.336 & $(0.141-0.771)$ & $0.011^{*}$ \\
Operation time & 0.003 & 0.008 & 0.415 & 1.003 & $(0.988-1.020)$ & 0.678 \\
\hline $\begin{array}{l}\text { Intercept is omitted. } \\
*<0.05 \mathrm{Cl} \text {, confidence interval; OR, odds ratio; RSV: subclinical right varicocele; USVG: ultrasound varicocele grading. }\end{array}$
\end{tabular}

(a)

\begin{tabular}{|c|c|c|c|}
\hline & Baseline & 3-mo & P \\
\hline Sperm Concentration $(\mathbf{n} \times \mathbf{1 0} / \mathrm{ml})$ & $15.2 \pm 20.3$ & $22.6 \pm 30$ & $0.002^{*}$ \\
\hline Progressive Motility (\%) & $25.1 \pm 16.8$ & $29.9 \pm 19.5$ & $0.011^{*}$ \\
\hline Normal Sperm Morphology (\%) & $0.7 \pm 1.7$ & $1.1 \pm 2.1$ & $0.024^{*}$ \\
\hline
\end{tabular}

(b)
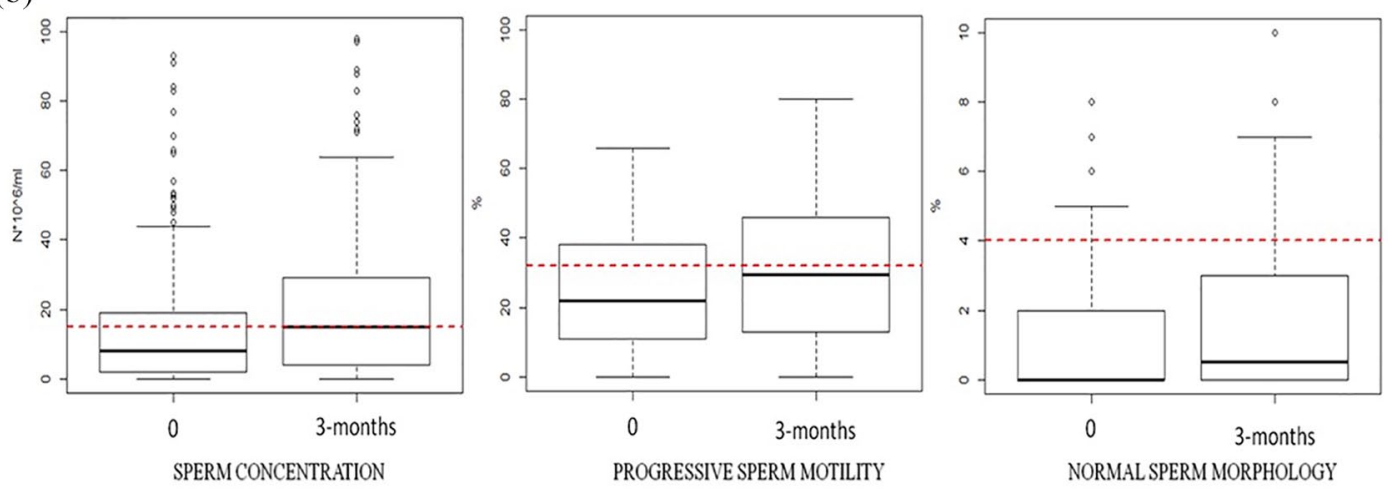

Figure 1. (a) comparison of semen parameters before and 3 months after microsurgical subinguinal varicocele repair. (b) box plots indicate quantitative changes in median semen parameters 3 months after surgery.

Please note, the red dotted lines indicate WHO reference values (sperm concentration $15 \times 10^{6} / \mathrm{ml}$; progressive sperm motility $32 \%$; normal sperm morphology $4 \%$ ).

\section{Discussion}

The aim of our study was to assess clinical factors affecting semen improvement in a large cohort of patients who underwent MSV, in a real life setting. We found a benefit from MSV in terms of mean SC improvement in older patients and in those with a higher USVG. Of clinical importance, we found the presence of concomitant RSV to negatively impact semen improvement after surgery.

Our interest was fueled by the fact that there is currently insufficient outcome data to support a formal evidence-based guideline, and that the evidence used to provide recommendations is, in general, of a low-quality. In this context, guidelines from the most important societies, including the American Society for Reproductive Medicine (ASRM), American Urological Association (AUA), European Academy of Andrology (EAA), and European Association of Urology (EAU) have in general, had an undefined impact on the current practice of varicocele treatment and management, giving unclear and sometimes contrasting indications. ${ }^{5-8}$ The reason for this has been attributed to the lack of well-designed studies and conflicting data regarding the impact of varicocele formation on infertility, abnormal semen 
(a)

\begin{tabular}{|c|c|c|c|}
\hline & $<35$ year-old & 235 year-old & $\mathbf{P}$ \\
\hline Patients (n) & 114 & 114 & - \\
\hline USG 1 (\%) & 7 & 15.4 & - \\
\hline USG 2 (\%) & 46.5 & 41.3 & - \\
\hline USG $3(\%)$ & 46.5 & 43.3 & - \\
\hline $\mathrm{SC}( \pm \mathrm{SD})\left(\mathrm{n} \times 10^{6} / \mathrm{ml}\right)$ & $13.7 \pm 18$ & $16.6 \pm 22.3$ & 0.830 \\
\hline PSM ( $($ SD) (\%) & $27.7 \pm 17.4$ & $22.5 \pm 15.8$ & 0.069 \\
\hline NSM ( $( \pm S D)(\%)$ & $1.3 \pm 2.5$ & $1.4 \pm 3.2$ & 0.767 \\
\hline
\end{tabular}

(b)
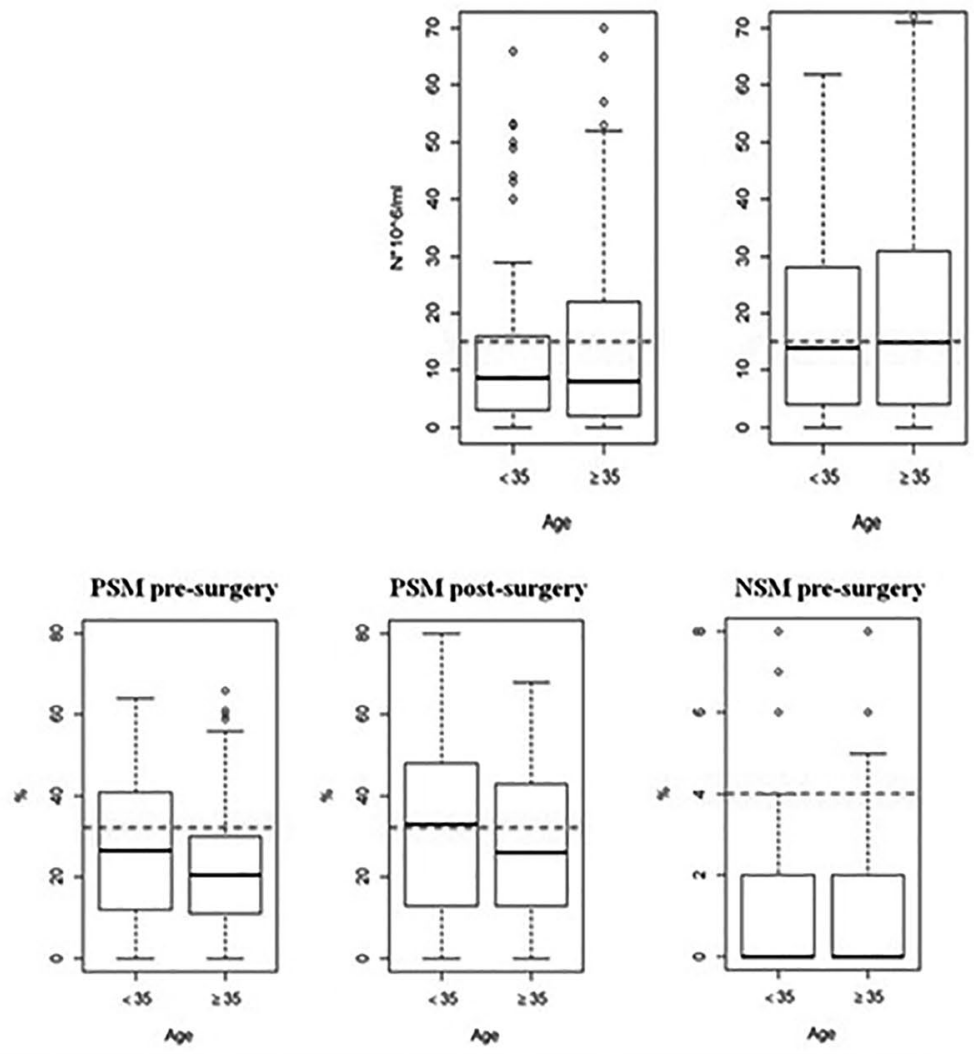

NSM post-surgery

Abbreviations used: SD, Standard Deviation; USG, Ultrasound Grading; SC, Sperm Concentration; PSM, Progressive Sperm Motility; NSM, Normal Sperm Morphology.

Figure 2. (a) Differences between $<35$ and $\geqslant 35$ year-old patients before surgery. (b) Box plots: quantitative changes in semen parameters 3 months after surgery in different age groups.

Please note, the red dotted lines indicate WHO reference values (sperm concentration $15 \times 10^{6} / \mathrm{ml}$; progressive sperm motility $32 \%$; normal sperm morphology $4 \%$ ).

parameters, decreased pregnancy rates, and the results of varicocele treatment. ${ }^{14}$

According to the EAU, the diagnosis of varicocele must be made by clinical examination and should be confirmed by US investigation and color Duplex analysis. ${ }^{11}$ Varicocelectomy should be performed in case of oligozoospermia and evidence of progressive testicular dysfunction, while a subclinical varicocele should not be treated. ${ }^{7}$ However, the AUA does not suggest imaging studies for standard evaluation unless the 
(a)

\begin{tabular}{|c|c|c|c|c|}
\hline & USVG & Pre-surgery & 3-Months & $P$ \\
\hline \multirow{2}{*}{$\begin{array}{c}\mathrm{SC}( \pm \mathrm{SD}) \\
\left(\mathrm{n} \times 10^{6} / \mathrm{mL}\right)\end{array}$} & USVG $_{1 \cdot 2}$ & $17.5 \pm 21.5$ & $24.9 \pm 28.3$ & $0.045^{*}$ \\
\hline & USVG $_{3}$ & $13.5 \pm 20.1$ & $20.8 \pm 33.1$ & $0.020^{*}$ \\
\hline \multirow{2}{*}{$\begin{array}{c}\operatorname{PSM}( \pm S D) \\
(\%)\end{array}$} & USVG $_{1 \cdot 2}$ & $24.5 \pm 16.1$ & $29.26 \pm 18.9$ & 0.166 \\
\hline & USVG $_{3}$ & $27.2 \pm 17.7$ & $31.1 \pm 20.3$ & 0.235 \\
\hline \multirow{2}{*}{$\begin{array}{c}\operatorname{NSM}( \pm S D) \\
(\%)\end{array}$} & USVG $_{1 \cdot 2}$ & $1.2 \pm 2.3$ & $1.7 \pm 2.4$ & $0.017^{*}$ \\
\hline & USVG $_{3}$ & $1.5 \pm 3.5$ & $1.7 \pm 3.3$ & 0.658 \\
\hline
\end{tabular}

(b)
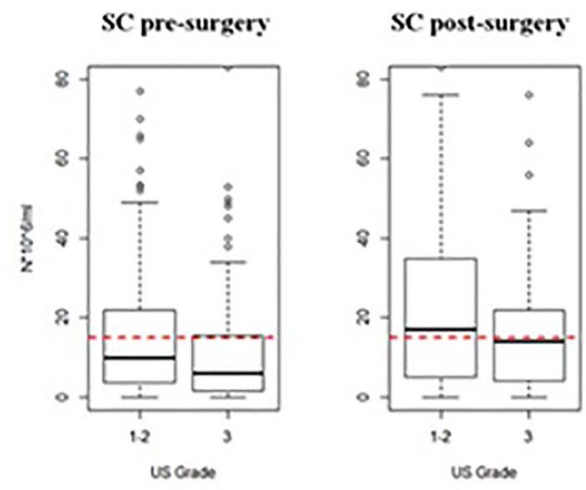

PSM pre-surgery

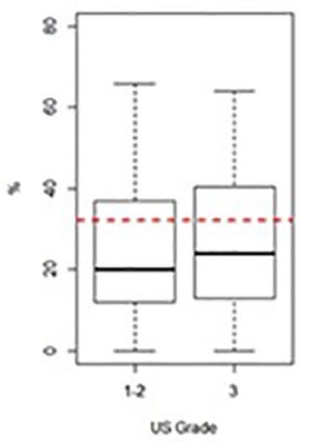

PSM post-surgery

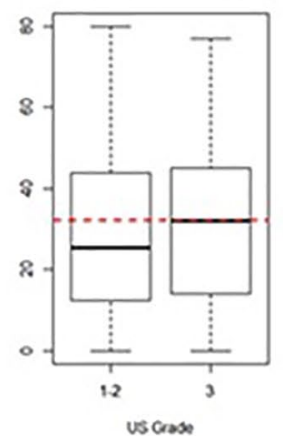

NSM pre-surgery

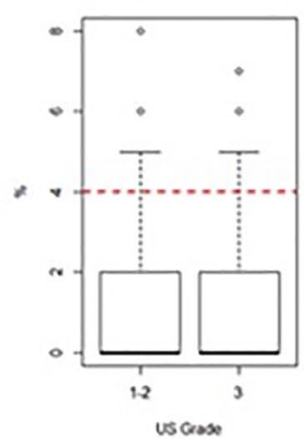

NSM post-surgery

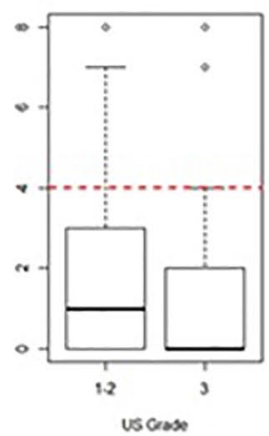

Abbreviations used:SD, Standard Deviation; USVG, Ultrasound Varicocele Grading; SC, Sperm Concentration; PSM, Progressive Sperm Motility; NSM, Normal Sperm Morphology.

Figure 3. (a) Comparison of semen parameters changes between US grade I-II and III after microsurgical subinguinal varicocelectomy. (b) Box plots: qualitative changes in median semen values 3 months after surgery in different US grade groups.

Please note, the red dotted lines indicate WHO reference values (sperm concentration $15 \times 106 / \mathrm{ml}$; progressive motility $32 \%$; normal forms $4 \%$ ).

physical exam is inconclusive. ${ }^{6}$ Scrotal US, however, may be indicated for clarification of an inconclusive physical examination of the scrotum. Spermatic venography may be useful to demonstrate the anatomic position of refluxing spermatic veins that recur or persist after varicocele repair.

In addition, the EAA underlines contradicting evidence that allows no reliable recommendation 
(a)

\begin{tabular}{|c|c|c|c|c|}
\hline & Varicocele Side & Pre-surgery & 3-Months & $\mathbf{P}$ \\
\hline \multirow{2}{*}{$\begin{array}{c}\mathrm{SC}( \pm \mathrm{SD}) \\
\left(\mathrm{n} \times 10^{6} / \mathrm{mL}\right)\end{array}$} & Left only & $15.04 \pm 18.9$ & $24.7 \pm 31.8$ & $<0.001^{* * *}$ \\
\hline & RSV & $15.9 \pm 26.8$ & $12.1 \pm 15.5$ & 0.848 \\
\hline \multirow{2}{*}{$\begin{array}{c}\text { PSM ( } \pm \text { SD) } \\
(\%)\end{array}$} & Left only & $24.01 \pm 17.04$ & $29.9 \pm 19.9$ & $0.006^{* *}$ \\
\hline & RSV & $28.7 \pm 15.9$ & $28.8 \pm 18.2$ & 0.839 \\
\hline \multirow[t]{2}{*}{$\begin{array}{c}\text { NSM ( } \pm \text { SD) } \\
(\%)\end{array}$} & Left only & $1.4 \pm 3.02$ & $1.8 \pm 2.8$ & $0.044^{*}$ \\
\hline & RSV & $1.02 \pm 1.9$ & $1.6 \pm 3.2$ & 0.292 \\
\hline
\end{tabular}

(b)
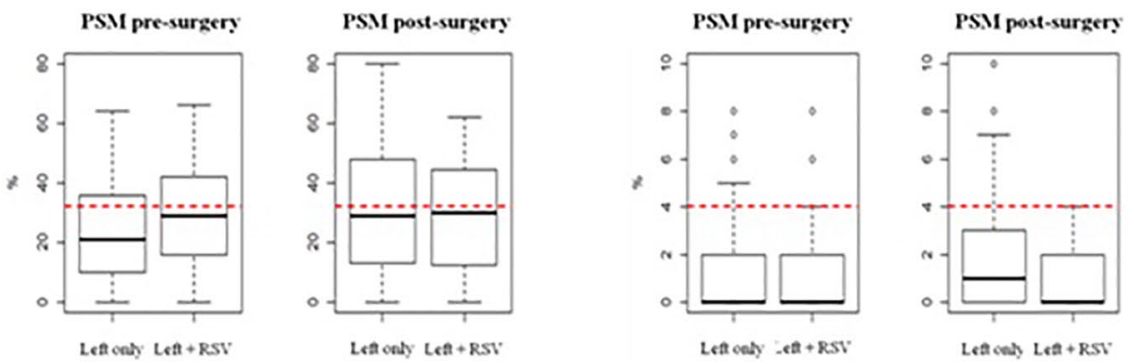

\footnotetext{
Abbreviations used: SD, Standard Deviation; RSV, Right Subclinical Varicocele; SC, Sperm Concentration; PSM, Progressive Sperm Motility; NSM, Normal Sperm Morphology.

${ }^{*}<0.05 ;^{* *}<0.01 ;{ }^{* * *}<0.001$
}

Figure 4. (a) Comparison of semen parameter changes after left microsurgical subinguinal varicocele repair in patients with or without right subclinical varicocele. (b) Box plots indicate quantitative changes in median semen parameters 3 months after surgery among all groups.

Please note, the red dotted lines indicate WHO reference values $\left(\right.$ sperm concentration $15 \times 10^{6} / \mathrm{ml}$; progressive sperm motility $32 \%$; normal sperm forms $4 \%$ ).

in favor for or against varicocelectomy. ${ }^{8}$ Varicocelectomy in infertile men with oligo-asthenoteratozoospermia and palpable varicocele should be discussed individually with the couple, while monitoring is suggested only for subclinical disease. ${ }^{8}$ However, scrotal US is considered part of a routine patient investigation. ${ }^{8,15}$ The Practice Committee of the ASRM is a 21-person committee with male reproductive urologists, andrologists and reproductive endocrinology, and infertility specialists. ${ }^{5}$ Their report provides a comprehensive overview of varicocele detection 
and management recommendations based on literature reviews and expert opinion. ${ }^{5}$ They suggest that the recommendations should be regarded as 'appropriate management' but not necessarily 'the only standard of practice'. ${ }^{5}$

The role of ultrasonography remains controversial because subclinical varicoceles have a poor concordance with those detected on physical examination. ${ }^{16}$ The WHO Manual for the Standardized Investigation, Diagnosis, and Management of the Infertile Male is cited as the source for varicocele confirmation by color Duplex US, however, its dogmatic emphasis on further investigation of grade I and subclinical varicoceles by US and thermography is not representative of the other previously mentioned reports. ${ }^{11}$

Previous studies examining clinical factors that possibly minimize semen improvement after varicocele repair have focused mainly on patient age and clinical grade. In present study, mean SC improved after MSV in $\geqslant 35$ year-old patients $(p=0.01)$. Similarly, Yazdani and colleagues reported statistically significant SC improvement after varicocelectomy in older patients, in this case, older than 30 years $(p<0.001) .{ }^{17}$ In addition, Ishikawa and colleagues found improvement of semen characteristics after varicocele repair in patients aged 20-29 years, and those over 30 years, concluding that it is reasonable to perform ligation to improve the semen characteristics in patients older than 40 years old. ${ }^{18}$ In addition, Cox regression analysis performed by Zhang and colleagues in a prospective cohort study of 120 males with varicocele who underwent MSV showed no role of age as a predictor of spontaneous pregnancy rate improvement. ${ }^{19}$ Of interest, Kimura and colleagues revealed that patients younger than 37 years old experienced greater early ( 3 months) and late ( $\geqslant 6$ months) improvements in semen parameters after microsurgical varicocele repair, however, they did not exclude the possibility of semen parameter improvement in older men, and concluded that microsurgical varicocele repair could also be beneficial for older men. ${ }^{20}$

The main problem concerning investigations on how much age can influence seminal parameters and their possible improvement after a treatment lies in the fact that the patient's age at the time of varicocele diagnosis is not related to the onset of the disease but is related to the patient's desire for children, this may represent a huge bias with no solution. In this context, what is of clinical importance is the effective improvement of the seminal parameters of these patients following surgery.

Contrasting findings have been reported by studies assessing the role of varicocele grade in a series of patients who had undergone surgery for infertility. ${ }^{21-24}$ Our data demonstrated that patients with $\mathrm{USVG}_{3}$ presented a three-times greater probability of SC improvement compared with patients with a lower USVG $(p=0.041)$. Our results were similar to Steckel's findings, where a greater relative improvement in sperm count in patients with a grade III varicocele than men with grade II or I disease was shown. ${ }^{21}$ However, Wang and colleagues reported that preoperative varicocele grade might not predict postoperative semen changes regardless of the possible existence of anatomic and ultrasonographic associations. $^{22}$ In addition, Braedel and colleagues ${ }^{23}$ found less improvement in sperm count in men with grade III varicocele than in men with smaller varicoceles. In addition, in a small cohort of patients divided according to the varicocele clinical grade, who all underwent a scrotal sonography with the Valsalva maneuver and showed pampiniform vein diameters $>3 \mathrm{~mm}$, Vahidi and colleagues highlighted an improved effect of microsurgical varicocelectomy on patients with a higher grade. ${ }^{24}$

A question to be resolved when considering treatment of varicocele is the gray area of subclinical concern, which relies on the diagnostic criteria employed..$^{25}$ Obviously, the degree of varicocele by palpation is subjective, but this dilemma apparently cannot be overcome by ultrasound, whose main characteristic is being operator-dependent. In addition, the heterogeneity and nonstandardization of the various scores and ultrasound classifications complicates the topic.

Our logistic regression model showed the probability of SC improvement was $75 \%$ lower when RSV was present $(p=0.11)$, which makes a contribution to the semen impairment. Although the boundary between clinical and subclinical varicocele remains elusive and no surgical recommendation has been given for the treatment of subclinical varicocele, ${ }^{5-8}$ several studies suggest a role in male infertility that supports our results. ${ }^{26-30}$ In a 2018 retrospective review on 190 infertile men who underwent a microsurgical varicocele repair, no differences were found in total motile 
sperm count improvement between men with clinical and subclinical varicoceles $(p=0.66) .{ }^{26}$

Even if no statistically significant differences were found for SC, as found in our data, in a well-designed meta-analysis of four randomized controlled trials, including 637 cases with left clinical and RSV ( 318 cases in the bilateral varicocelectomy group and 319 cases in the left varicocelectomy group), the fixed effects model combined difference in sperm PSM between the two groups was $6.42 \%$ (CI $95 \%$ 5.09-7.75), the random effects model combined difference in NSM between the two groups was $2.04 \%$ (CI 95\% 0.60-3.48). ${ }^{27}$ In addition to this, the odds ratio shown by the fixed effects model in spontaneous pregnancy rate was 1.73 (CI 95\% 1.24-2.43) indicating bilateral varicocelectomy may be superior to unilateral surgery for infertile male patients with left clinical and RSV. ${ }^{27}$ Despite not increasing pregnancy rates, metaanalysis performed by Kim and colleagues showed that surgical repair for subclinical varicocele could improve forward sperm PSM, ${ }^{28}$ although no statistically significant benefit derived from surgery when SC, in contrast to our results, was considered. Consistent with our conclusions, and despite the different technique used, in a prospective study reported by Cantoro and colleagues percutaneous embolization was performed in 218 male patients with left subclinical varicocele and abnormal sperm parameters, the control group included 119 cases. Afteralong follow-up (39.4 \pm 6.5 months), the mean SC and total motility in the treatment group were better than those in the control group, indicating that subclinical varicocele should be diagnosed and treated. ${ }^{29}$ In addition, Cervellion and colleagues revealed that subclinical varicocele can progress to clinical varicocele in $28 \%$ of male children. Although there is no related study in adults men, bilateral varicocelectomy might stop the progress of subclinical varicocele in infertile men..$^{30}$ In light of this, a recent study suggested that subclinical varicocele might be a less severe form of clinical varicocele and the pathogenic mechanism was the same, justifying its treatment. ${ }^{31}$

Because varicocele-related subfertility is a condition with a ill-defined pathophysiology, and because is has not yet been clarified how clinical factors such as patient age, varicocele grade, and the presence of a concomitant RSV may influence semen parameters, the current challenges in clinical practice lie in determining when surgery should be performed and for which patients. In our study, we observed the clinical outcome using seminal improvements as the primary determinant factor in a large cohort. This study helped to reveal the most appropriate treatment strategies for infertile men with varicocele, indicating a benefit from surgery also in older patients and for those with higher grade disease. In addition, our results suggest that bilateral varicocelectomy should be considered in patients with left clinical and RSV.

Several limitations to this study should be acknowledged, of which the single institution design is probably the most relevant, and has the potential to result in a sample of patients that is not representative of the general population. However, given that the reference department is a tertiary referral center for the city of Barcelona and for surrounding cities and the region of Catalonia (Spain), we believe it is safe to assume that this limitation was minimal in this study. Another limitation of the present study lies in the short follow-up period (3 months). However, current evidence indicates that the degree of improvement in semen parameters 3 months after varicocelectomy remains the same, or stable, 12 months after surgery. ${ }^{20-32}$ In addition, no comparison with other varicocelectomy techniques was performed, with microsurgical varicocele repair being the gold standard in varicocele treatment. ${ }^{7}$ Another limitation of the current study is its retrospective design for which we did not have data collection with standardized protocols regarding the hormonal profiles of the patients, therefore, making any speculation impossible. Similarly, because the quantification of the clinical disease was missing for a large percentage of the patients, we only used US grading in the analysis. Because of this, physical examination is often ambiguous due to its subjective nature and dependence on the experience of the examiner. It is of limited value in very obese patients, patients with high-located testes, patients with a history of surgery in the scrotal or groin region, patients with a coexistent hydrocele, and in patients with a postoperative recurrence of varicocele. Under these conditions, US assessment may be very helpful or even necessary in these patients.

Despite this, our results promote MSV as an effective technique for the treatment of varicocele-related infertility, even in older patients and those with a 
higher US grade. In addition, it highlights the role that RSV plays in semen improvement after MSV.

\section{Conclusion}

When surgical treatment in considered in infertile men with varicocele, MSV should be offered for older patients. Patients with higher USVG benefit from surgery in terms of postoperative semen changes. In infertile men with both RSV and a left clinical disease, a bilateral varicocele repair should be considered.

\section{Authors' note}

Riccardo Ievoli is now affiliated to Department of Economics and Management, University of Ferrara, Ferrara, Italy.

\section{Acknowledgements}

This retrospective study was conducted according to the guidelines and principles of the Declaration of Helsinki and standard ethical conduct for research involving humans; after approval of the our Ethical Committee for Clinical Research, all patients signed an informed consent agreeing to supply their own anonymous data for this and future studies. The study also guaranteed compliance at all times with Law 15/1999 on Protection of Personal Data (Spanish Government).

The authors thank Dana Kuefner PhD for reviewing the language in this manuscript.

F.P. and D.M.M. contributed equally to this work. Conceptualization: F.P., D.M.M., J.S.G., J.S.C. Data curation: F.P., D.M.M., J.S.C. Formal analysis: F.P., R.I. Investigation: F.P., D.M.M., R.I., G.V.M.d.S., C.G.S., J.F.V.O., M.F.P.G., A.V.S., J.S.G., J.S.C. Methodology: F.P., R.I., C.G.S., J.F.V.O., J.S.C. Supervision: L.B.A., E.M., E.R.C., J.S.C. Validation: D.M.M., M.F.P.G., A.V.S., J.S.G., J.S.C. Writing - original draft: F.P., G.V.M.d.S. Writing - review \& editing: F.P., G.V.M.d.S., J.S.C.

\section{Funding}

The author(s) received no financial support for the research, authorship, and/or publication of this article.

\section{Conflict of interest statement}

The authors declare that there is no conflict of interest.
ORCID iD

Franco Palmisano (D) https://orcid.org/0000-0002 -0197-9033

\section{References}

1. World Health Organization. The influence of varicocele on parameters of fertility in a large group of men presenting to infertility clinics. Fertil Steril 1992; 57: 1289.

2. Agarwal A, Deepinder F, Cocuzza M, et al. Efficacy of varicocelectomy in improving semen parameters: new meta-analytical approach. Urology 2007; 70: 532-538.

3. Will MA, Swain J, Fode M, et al. The great debate: varicocele treatment and impact on fertility. Fertil Steril 2011; 95: 841-852.

4. Pallotti F, Paoli D, Carlini T, et al. Varicocele and semen quality: a retrospective case-control study of 4230 patients from a singlecentre. $\mathcal{F}$ Endocrinol Invest 2018; 41: 185-192.

5. American Society for Reproductive Medicine. Report on varicocele and infertility: a committee opinion. Fertil Steril 2014; 102: 1556-1560.

6. American Urological Association. The optimal evaluation of the infertile male: $A U A$ best practice statement. Linthicum, MD: American Urological Association Education and Research Inc; 2010.

7. Jungwirth A, Diemer T, Kopa Z, et al. European association of urology guidelines on male infertility. In: Presented at the EAU Annual Congress Copenhagen 2018. Arnhem, The Netherlands: EAU Guidelines Office.

8. Colpi GM, Francavilla S, Haidl G, et al. European Academy of Andrology guideline Management of oligo-astheno-teratozoospermia. Andrology 2018; 6: 513-524.

9. Ding H, Tian J, Du W, et al. Open nonmicrosurgical, laparoscopic or open microsurgical varicocelectomy for male infertility: a metaanalysis of randomized controlled trials. BfU Int 2012; 110: 1536-1542.

10. Cooper TG, Noonan E, von Eckardstein S, et al. World Health Organization reference values for human semen characteristics. Hum Reprod Update 2010; 16: 231-245.

11. Rowe PJ, Comhaire FH, Hargreave TB, et al.; World Health Organization. WHO manual for the standardized investigation, diagnosis, and management of the infertile male. New York: Cambridge; 2000. 
12. Nelder JA and Wedderburn RWM. Generalized linear models. F R Stat Soc A 1972; 135: 370384.

13. Hosmer Jr DW, Lemeshow $\mathrm{S}$ and Sturdivant RX. Applied logistic regression. Vol. 398. John Wiley \& Sons, 2013.

14. Shridharani A, Owen RC, Elkelany OO, et al. The significance of clinical practice guidelines on adult varicocele detection and management. Asian f Androl 2016; 18: 269-275.

15. Lotti $F$ and Maggi M. Ultrasound of the male genital tract in relation to male reproductive health. Hum Reprod Update 2015; 21: 56-83.

16. Petros JA, Andriole GL, Middleton WD, et al. Correlation of testicular color Doppler ultrasonography, physical examination and venography in the detection of left varicoceles in men with infertility. F Urol 1991; 145: 785-788.

17. Yazdani M, Hadi M, Abbasi H, et al. Efficacy of varicocele repair in different age groups. Urology 2015; 86: 273-275.

18. Ishikawa $T$ and Fujisawa $M$. Effect of age and grade on surgery for patients with varicocele. Urology 2005; 65: 768-772.

19. Zhang JW, Xu QQ, Kuang YL, et al. Predictors for spontaneous pregnancy after microsurgical subinguinal varicocelectomy: a prospective cohort study. Int Urol Nephrol 2017; 49: 955-960.

20. Kimura M, Nagao K, Tai T, et al. Age is a significant predictor of early and late improvement in semen parameters after microsurgical varicocele repair. Andrologia 2017; 49.

21. Steckel J, Dicker AP and Goldstein M. Relationship between varicoceles size and response to varicocelectomy. $\mathcal{F}$ Urol 1993; 149: 769-771.

22. Wang H, Wang X, Fu D, et al. Does varicocele grade predict the postoperative changes of semen parameters following left inguinal microvaricocelectomy? Asian f Urol 2015; 2: 163-166.
23. Braedel HU, Steffens J, Ziegler M, et al. A possible ontogenetic etiology for idiopathic left varicocele. F Urol 1994; 151: 62-66.

24. Vahidi S, Moein M, Nabi A, et al. Effects of microsurgical varicocelectomy on semen analysis and sperm function tests in patients with different grades of varicocele: role of sperm functional tests in evaluation of treatments outcome. Andrologia 2018; 50: e13069.

25. Howards SS. Subclinical varicocele. Fertil Steril 1992; 57: 725-726.

26. Thirumavalavan N, Scovell JM, Balasubramanian A, et al. The impact of microsurgical repair of subclinical and clinical varicoceles on total motile sperm count: is there a difference? Urology 2018; 120: 109-113.

27. Niu Y, Wang D, Chen Y, et al. Comparison of clinical outcome of bilateral and unilateral varicocelectomy in infertile males with left clinical and right subclinical varicocele: A meta-analysis of randomised controlled trials. Andrologia 2018; 50: e13078.

28. Kim HJ, Seo JT, Kim KJ, et al. Clinical significance of subclinical varicocelectomy in male infertility: systematic review and metaanalysis. Andrologia 2016; 48: 654-661.

29. Cantoro U, Polito M and Muzzonigro G. Reassessing the role of subclinical varicocele in infertile men with impaired semen quality: a prospective study. Urology 2015; 85: 826-830.

30. Cervellione RM, Corroppolo $\mathrm{M}$ and Bianchi A. Subclinical varicocele in the pediatric age group. f Urol 2008; 179: 717-719; discussion 719 .

31. Chen SS. Significant predictive factors for subfertility in patients with subclinical varicocele. Andrologia 2017; 49.

32. Fukuda T, Miyake H, Enatsu N, et al. Assessment of time-dependent changes in semen parameters in infertile men after microsurgical varicocelectomy. Urology 2015; 86: 48-51.
Visit SAGE journals online journals.sagepub.com/ home/tau

(SAGE journals 